\title{
Solutions to a boundary value problem of a fourth-order impulsive differential equation
}

Jingli Xie' and Zhiguo Luo ${ }^{2 *}$

"Correspondence:
luozg@hunnu.edu.cn
${ }^{2}$ Department of Mathematics,
Hunan Normal University,
Changsha, Hunan 410081, P.R. China
Full list of author information is
available at the end of the article

available at the end of the article

\begin{abstract}
This paper is concerned with the existence of solutions to a boundary value problem of a fourth-order impulsive differential equation with a control parameter $\lambda$. By employing some existing critical point theorems, we find the range of the control parameter in which the boundary value problem admits at least one solution. It is also shown that under certain conditions there exists an interval of the control parameter in which the boundary value problem possesses infinitely many solutions. The main results are also demonstrated with examples.
\end{abstract}

MSC: 34B15; 34B18; 34B37; 58E30

Keywords: critical point theorem; impulsive differential equations; boundary value problem

\section{Introduction}

Fourth-order two-point boundary value problems of ordinary differential equations are widely employed by engineers to describe the beam deflection with two simply supported ends [1-3]. One example is the following fourth-order two-point boundary value problem:

$$
\left\{\begin{array}{l}
u^{(i v)}(t)+A u^{\prime \prime}(t)+B u(t)=\lambda f(t, u(t)), \quad t \in[0,1] \\
u(0)=u(1)=u^{\prime \prime}(0)=u^{\prime \prime}(1)=0
\end{array}\right.
$$

where $u^{(i v)}(t), u^{\prime \prime \prime}(t), u^{\prime \prime}(t)$ are the fourth, third, and second derivatives of $u(t)$ with respect to $t$, respectively, $f \in C([0,1] \times R, R), A$ and $B$ are two real constants. System (1.1) has been studied in [4-7] and the references therein. For a beam, $t=0$ and $t=1$ in (1.1) refer to the two ends of the beam. At other locations of the beam, $t \in(0,1)$, there may be some sudden changes in loads placed on the beam, or some unexpected forces working on the beam. These sudden changes may result in impulsive effects for the governing differential equation. This motivates us to consider the following boundary value problem for a fourth-order impulsive differential equation:

$$
\left\{\begin{array}{l}
u^{(i v)}(t)+A u^{\prime \prime}(t)+B u(t)=\lambda f(t, u(t)), \quad t \neq t_{j}, t \in[0,1], \\
\Delta u^{\prime \prime}\left(t_{j}\right)=I_{1 j}\left(u^{\prime}\left(t_{j}\right)\right), \quad-\Delta u^{\prime \prime \prime}\left(t_{j}\right)=I_{2 j}\left(u\left(t_{j}\right)\right), \quad j=1,2, \ldots, m, \\
u(0)=u(1)=u^{\prime \prime}(0)=u^{\prime \prime}(1)=0,
\end{array}\right.
$$


where $I_{1 j}, I_{2 j} \in C(R, R), 0=t_{0}<t_{1}<t_{2}<\cdots<t_{m}<t_{m+1}=1$, and the operator $\Delta$ is defined as $\Delta U\left(t_{j}\right)=U\left(t_{j}^{+}\right)-U\left(t_{j}^{-}\right)$, where $U\left(t_{j}^{+}\right)\left(U\left(t_{j}^{-}\right)\right)$denotes the right-hand (left-hand) limit of $U$ at $t_{j}$ and $\lambda>0$ is referred to as a control parameter.

We are mainly concerned with the existence of solutions of system (1.2). A function $u(t) \in C([0,1])$ is said to be a (classical) solution of $(1.2)$ if $u(t)$ satisfies (1.2). In literature, tools employed to establish the existence of solutions of impulsive differential equations include fixed point theorems, the upper and lower solutions method, the degree theory, critical point theory and variational methods. See, for example, [8-20]. In this paper, we establish the existence of solutions of (1.2) by converting the problem to the existence of critical points of some variational structure. In this paper we regard $\lambda$ as a parameter and find the ranges in which (1.2) admits at least one and infinitely many solutions, respectively. Note that when $\lambda=1$ system (1.2) reduces to the one studied in [21]. Our results extend those ones in [21].

The rest of this paper is organized as follows. In Section 2 we present some preliminary results. Our main results and their proofs are given in Section 3.

\section{Preliminaries}

Throughout we assume that $A$ and $B$ satisfy

$$
A \leq 0 \leq B .
$$

Let

$$
H_{0}^{1}([0,1])=\left\{u \in L^{2}([0,1]): u^{\prime} \in L^{2}([0,1]), u(0)=u(1)=0\right\},
$$

and

$$
H^{2}([0,1])=\left\{u \in L^{2}([0,1]): u^{\prime}, u^{\prime \prime} \in L^{2}([0,1])\right\} .
$$

Take $X:=H^{2}([0,1]) \cap H_{0}^{1}([0,1])$ and define

$$
\|u\|_{X}=\left(\int_{0}^{1}\left(\left|u^{\prime \prime}(t)\right|^{2}-A\left|u^{\prime}\right|^{2}+B|u|^{2}\right) d t\right)^{\frac{1}{2}}, \quad u \in X .
$$

Since $A$ and $B$ satisfy (2.1), it is straightforward to verify that (2.2) defines a norm for the Sobolev space $X$ and this norm is equivalent to the usual norm defined as follows:

$$
\|u\|=\left(\int_{0}^{1} u^{\prime \prime}(t)^{2} d t\right)^{\frac{1}{2}} .
$$

It follows from (2.1) that

$$
\|u\| \leq\|u\|_{X} .
$$

For the norm in $C^{1}([0,1])$,

$$
\|u\|_{\infty}=\max \left(\max _{t \in[0,1]}|u(t)|, \max _{t \in[0,1]}\left|u^{\prime}(t)\right|\right),
$$

we have the following relation. 
Lemma 2.1 Let $M_{1}=1+\frac{1}{\pi}$. Then $\|u\|_{\infty} \leq M_{1}\|u\|_{X}, \forall u \in X$.

Proof The proof follows easily from Wirtinger's inequality [22], Lemma 2.3 of [23] and Hölder's inequality. The detailed argument is similar to the proof of Lemma 2.2 in [21], and we thus omit it here.

Define a functional $\varphi_{\lambda}$ as

$$
\varphi_{\lambda}(u)=\Phi(u)-\lambda \Psi(u), \quad u \in X,
$$

where

$$
\Phi(u)=\frac{1}{2}\|u\|_{X}^{2}+\sum_{j=1}^{m} \int_{0}^{u^{\prime}\left(t_{j}\right)} I_{1 j}(s) d s+\sum_{j=1}^{m} \int_{0}^{u\left(t_{j}\right)} I_{2 j}(s) d s
$$

and

$$
\Psi(u)=\int_{0}^{1} F(t, u) d t
$$

with

$$
F(t, u)=\int_{0}^{u(t)} f(t, s) d s
$$

Note that $\varphi_{\lambda}$ is Fréchet differentiable at any $u \in X$, and for any $v \in X$ we have

$$
\begin{aligned}
\varphi_{\lambda}^{\prime}(u)(v)= & \lim _{h \rightarrow 0} \frac{\varphi_{\lambda}(u+h v)-\varphi_{\lambda}(u)}{h} \\
= & \int_{0}^{1}\left(u^{\prime \prime}(t) v^{\prime \prime}(t)-A u^{\prime}(t) v^{\prime}(t)+B u(t) v(t)\right) d t+\sum_{j=1}^{m} I_{2 j}\left(u\left(t_{j}\right)\right) v\left(t_{j}\right) \\
& +\sum_{j=1}^{m} I_{1 j}\left(u^{\prime}\left(t_{j}\right)\right) v^{\prime}\left(t_{j}\right)-\lambda \int_{0}^{1} f(t, u(t)) v(t) d t .
\end{aligned}
$$

Next we show that a critical point of the functional $\varphi_{\lambda}$ is a solution of system (1.2).

Lemma 2.2 If $u \in X$ is a critical point of $\varphi_{\lambda}$, then $u$ is a solution of system (1.2).

Proof Suppose that $u \in X$ is a critical point of $\varphi_{\lambda}$. Then for any $v \in X$ one has

$$
\begin{aligned}
\lambda \int_{0}^{1} f(t, u(t)) v(t) d t= & \int_{0}^{1}\left(u^{\prime \prime}(t) v^{\prime \prime}(t)-A u^{\prime}(t) v^{\prime}(t)+B u(t) v(t)\right) d t \\
& +\sum_{j=1}^{m} I_{2 j}\left(u\left(t_{j}\right)\right) v\left(t_{j}\right)+\sum_{j=1}^{m} I_{1 j}\left(u^{\prime}\left(t_{j}\right)\right) v^{\prime}\left(t_{j}\right) .
\end{aligned}
$$

For $j \in\{1,2, \ldots, m\}$, choose $v \in X$ such that $v(t)=0$ for $t \in\left[0, t_{j}\right] \cup\left[t_{j+1}, 1\right]$, then we have

$$
\int_{t_{j}}^{t_{j+1}}\left(u^{(i v)}+A u^{\prime \prime}(t)+B u(t)\right) v(t) d t=\lambda \int_{t_{j}}^{t_{j+1}} f(t, u(t)) v(t) d t
$$


Thus

$$
u^{(i v)}+A u^{\prime \prime}(t)+B u(t)=\lambda f(t, u(t)) \quad \text { a.e. } t \in\left(t_{j}, t_{j+1}\right) .
$$

Therefore, by (2.7) we have

$$
\sum_{j=1}^{m}\left(\Delta u^{\prime \prime \prime}\left(t_{j}\right)+I_{2 j}\left(u\left(t_{j}\right)\right)\right) v\left(t_{j}\right)-\sum_{j=1}^{m}\left(\Delta u^{\prime \prime}\left(t_{j}\right)-I_{1 j}\left(u^{\prime}\left(t_{j}\right)\right)\right) v^{\prime}\left(t_{j}\right)=0 .
$$

Next we show that $u$ satisfies

$$
-\Delta u^{\prime \prime \prime}\left(t_{j}\right)=I_{2 j}\left(u\left(t_{j}\right)\right), \quad j=1,2, \ldots, m .
$$

Suppose on the contrary that there exists some $j \in\{1,2, \ldots, m\}$ such that

$$
\Delta u^{\prime \prime \prime}\left(t_{j}\right)+I_{2 j}\left(u\left(t_{j}\right)\right) \neq 0 .
$$

Pick

$$
v(t)=\left(t^{3}-3 t_{j}^{2} t\right) \prod_{i=0, i \neq j}^{m+1}\left(\frac{1}{3} t^{3}-\frac{1}{2}\left(t_{j}+t_{i}\right) t^{2}+t_{j} t_{i} t+\frac{1}{6} t_{i}^{3}-\frac{1}{2} t_{j} t_{i}^{2}\right),
$$

then

$$
\begin{aligned}
v^{\prime}(t)= & 3\left(t^{2}-t_{j}^{2}\right) \prod_{i=0, i \neq j}^{m+1}\left(\frac{1}{3} t^{3}-\frac{1}{2}\left(t_{j}+t_{i}\right) t^{2}+t_{j} t_{i} t+\frac{1}{6} t_{i}^{3}-\frac{1}{2} t_{j} t_{i}^{2}\right) \\
& +\left(t^{3}-3 t_{j} t\right) \sum_{k=0, k \neq j}^{m+1}\left\{\left(t^{2}-\left(t_{k}+t_{j}\right) t+t_{k} t_{j}\right)\right. \\
& \left.\cdot \prod_{i=0, i \neq j, k}^{m+1}\left(\frac{1}{3} t^{3}-\frac{1}{2}\left(t_{j}+t_{i}\right) t^{2}+t_{j} t_{i} t+\frac{1}{6} t_{i}^{3}-\frac{1}{2} t_{j} t_{i}^{2}\right)\right\} .
\end{aligned}
$$

Clearly, $v \in X$. Simple calculations show that $v\left(t_{i}\right)=0, i=1,2, \ldots, j-1, j+1, \ldots, m+1, v\left(t_{j}\right) \neq$ 0 and $v^{\prime}\left(t_{i}\right)=0, i=1,2, \ldots, m+1$. Thus

$$
\frac{1}{3} t_{j}^{3}\left(\Delta u^{\prime \prime \prime}\left(t_{j}\right)+I_{2 j}\left(u\left(t_{j}\right)\right)\right) \prod_{i=0, i \neq j}^{m+1}\left(t_{i}-t_{j}\right)^{3}=0,
$$

which is a contradiction. Similarly, one can show that $\Delta u^{\prime \prime}\left(t_{j}\right)=I_{1 j}\left(u^{\prime}\left(t_{j}\right)\right), j=1,2, \ldots, m$. Therefore, $u$ is a solution of (1.2).

For $r_{1}, r_{2} \in \mathbb{R}$ with $r_{1}<r_{2}$, we define

$$
\alpha\left(r_{1}, r_{2}\right)=\sup _{v \in \Phi^{-1}\left(\left(r_{1}, r_{2}\right)\right)} \frac{\Psi(v)-\sup _{u \in \Phi^{-1}\left(\left(-\infty, r_{1}\right)\right)} \Psi(u)}{\Phi(v)-r_{1}}
$$


and

$$
\beta\left(r_{1}, r_{2}\right)=\inf _{v \in \Phi^{-1}\left(\left(r_{1}, r_{2}\right)\right)} \frac{\sup _{u \in \Phi^{-1}\left(\left(r_{1}, r_{2}\right)\right)} \Psi(u)-\Psi(v)}{r_{2}-\Phi(v)} .
$$

For $r \in \mathbb{R}$, we define

$$
\begin{aligned}
& \rho_{1}(r)=\inf _{v \in \Phi^{-1}((-\infty, r))} \frac{\sup _{u \in \Phi^{-1}((-\infty, r))} \Psi(u)-\Psi(v)}{r-\Phi(v)}, \\
& \rho_{2}(r)=\sup _{v \in \Phi^{-1}((r,+\infty))} \frac{\Psi(v)-\sup _{u \in \Phi^{-1}((-\infty, r])} \Psi(u)}{\Phi(v)-r} .
\end{aligned}
$$

\section{Main results}

\subsection{Existence of at least one solution}

In this section we derive conditions under which system (1.2) admits at least one solution. For this purpose, we introduce the following assumption.

(H1) Assume that there exist two positive constants $k_{1}$ and $k_{2}$ such that for each $u \in X$

$$
0 \leq \sum_{j=1}^{m} \int_{0}^{u^{\prime}\left(t_{j}\right)} I_{1 j}(s) d s \leq k_{1} \max _{j \in\{1,2, \ldots, m\}}\left|u^{\prime}\left(t_{j}\right)\right|^{2}
$$

and

$$
0 \leq \sum_{j=1}^{m} \int_{0}^{u\left(t_{j}\right)} I_{2 j}(s) d s \leq k_{2} \max _{j \in\{1,2, \ldots, m\}}\left|u\left(t_{j}\right)\right|^{2}
$$

Let $k_{0}=2-\frac{A}{6}+\frac{B}{60}$ and $k_{3}=k_{0}+k_{1}+\frac{1}{4} k_{2}$ with $k_{1}$ given in (3.1) and $k_{2}$ given in (3.2). For constants $c_{1}, c_{2}$, and $c$ satisfying

$$
c_{1}<\sqrt{2 k_{0}} M_{1} c<\sqrt{2 k_{3}} M_{1} c<c_{2}
$$

we define

$$
a\left(c_{2}, c\right)=\frac{\int_{0}^{1} \max _{|u| \leq c_{2}} F(t, u) d t-\int_{0}^{1} F\left(t, u_{1}(t)\right) d t}{c_{2}^{2}-2 k_{3} M_{1}^{2} c^{2}}
$$

and

$$
b\left(c_{1}, c\right)=\frac{\int_{0}^{1} F\left(t, u_{1}(t)\right) d t-\int_{0}^{1} \max _{|u| \leq c_{1}} F(t, u) d t}{2 k_{3} M_{1}^{2} c^{2}-c_{1}^{2}},
$$

where

$$
u_{1}(t)=c t(1-t)
$$

Note that for every $c>0$ and $t \in[0,1]$ we have $\left|u_{1}(t)\right|=c t(1-t) \leq \frac{c}{4}<c$. Since $A \leq$ $0 \leq B$, then $k_{0}>2$. Thus, if $c$ and $c_{2}$ satisfy (3.3), then $c_{2}>c$ and $\int_{0}^{1} \max _{|u| \leq c_{2}} F(t, u) d t-$ $\int_{0}^{1} F\left(t, u_{1}(t)\right) d t>0$ and hence $a\left(c_{2}, c\right)>0$. 
Theorem 3.1 Assume that $(\mathrm{H} 1)$ is satisfied. If there exist constants $c_{1}, c_{2}$, and c satisfying (3.3) and

$$
0<a\left(c_{2}, c\right)<b\left(c_{1}, c\right)
$$

then, for each $\lambda \in\left(\lambda_{1}, \lambda_{2}\right)$, system (1.2) admits at least one solution $u$ and $\|u\|_{X}<\frac{c_{2}}{M_{1}}$, where $\lambda_{1}=\frac{1}{2 M_{1}^{2} b\left(c_{1}, c\right)}$ and $\lambda_{2}=\frac{1}{2 M_{1}^{2} a\left(c_{2}, c\right)}$.

Proof By Lemma 2.2, it suffices to show the functional $\varphi_{\lambda}$ defined in (2.3) has at least one critical point. We prove this by verifying the conditions given in [10, Theorem 5.1]. Note that $\Phi$ defined in (2.4) is a nonnegative Gâteaux differentiable, coercive, and sequentially weakly lower semicontinuous functional, and its Gâteaux derivative admits a continuous inverse on $X^{*}$. Moreover, $\Psi$ defined in (2.5) is a continuously Gâteaux differentiable functional whose Gâteaux derivative is compact. Set

$$
r_{1}=\frac{c_{1}^{2}}{2 M_{1}^{2}}, \quad r_{2}=\frac{c_{2}^{2}}{2 M_{1}^{2}} .
$$

Note that $u_{1}(t)=c t(1-t) \in X$. It then follows from $(\mathrm{H} 1)$ that

$$
\begin{aligned}
\Phi\left(u_{1}\right) & =\frac{1}{2}\left\|u_{1}\right\|_{X}^{2}+\sum_{j=1}^{m} \int_{0}^{u_{1}^{\prime}\left(t_{j}\right)} I_{1 j}(s) d s+\sum_{j=1}^{m} \int_{0}^{u_{1}\left(t_{j}\right)} I_{2 j}(s) d s \\
& =\left(2-\frac{A}{6}+\frac{B}{60}\right) c^{2}+\sum_{j=1}^{m} \int_{0}^{c-2 c t_{j}} I_{1 j}(s) d s+\sum_{j=1}^{m} \int_{0}^{c t_{j}\left(1-t_{j}\right)} I_{2 j}(s) d s \\
& =k_{0} c^{2}+\sum_{j=1}^{m} \int_{0}^{c-2 c t_{j}} I_{1 j}(s) d s+\sum_{j=1}^{m} \int_{0}^{c t_{j}\left(1-t_{j}\right)} I_{2 j}(s) d s \\
& \leq k_{0} c^{2}+k_{1} \max _{j}\left|c-2 c t_{j}\right|^{2}+k_{2} \max _{j}\left|c t_{j}\left(1-t_{j}\right)\right|^{2} \\
& \leq k_{0} c^{2}+k_{1} c^{2}+\frac{1}{4} k_{2} c^{2} \\
& =k_{3} c^{2}
\end{aligned}
$$

and

$$
\Phi\left(u_{1}\right) \geq \frac{1}{2}\left\|u_{1}\right\|_{X}^{2}=k_{0} c^{2}
$$

By (3.3) we have

$$
r_{1}=\frac{c_{1}^{2}}{2 M_{1}^{2}}<k_{0} c^{2} \leq \Phi\left(u_{1}\right) \leq k_{3} c^{2}<\frac{c_{2}^{2}}{2 M_{1}^{2}}=r_{2} .
$$

For $u \in X$ satisfying $\Phi(u)<r_{2}$, by Lemma 2.1 , one has

$$
|u|^{2} \leq\|u\|_{\infty}^{2} \leq M_{1}^{2}\|u\|_{X}^{2} \leq 2 M_{1}^{2} \Phi(u)<2 M_{1}^{2} r_{2}=c_{2}^{2}, \quad t \in[0,1],
$$


which implies that

$$
\Psi(u)=\int_{0}^{1} F(t, u(t)) d t \leq \int_{0}^{1} \max _{|u| \leq c_{2}} F(t, u) d t .
$$

Hence

$$
\sup _{u \in \Phi^{-1}\left(\left(r_{1}, r_{2}\right)\right)} \Psi(u) \leq \sup _{u \in \Phi^{-1}\left(\left(-\infty, r_{2}\right)\right)} \Psi(u) \leq \int_{0}^{1} \max _{|u| \leq c_{2}} F(t, u) d t .
$$

For $u \in X$ with $\Phi(u)<r_{1}$, one can similarly obtain

$$
\sup _{u \in \Phi^{-1}\left(\left(-\infty, r_{1}\right)\right)} \Psi(u) \leq \int_{0}^{1} \max _{|u| \leq c_{1}} F(t, u) d t
$$

It follows from the definition of $\beta\left(r_{1}, r_{2}\right)$ that

$$
\beta\left(r_{1}, r_{2}\right) \leq \frac{\sup _{u \in \Phi^{-1}\left(\left(-\infty, r_{2}\right)\right)} \Psi(u)-\Psi\left(u_{1}\right)}{r_{2}-\Phi\left(u_{1}\right)} .
$$

Note that $\Phi\left(u_{1}\right)<r_{2}$. By (3.10) one has

$$
\beta\left(r_{1}, r_{2}\right) \leq \frac{\int_{0}^{1} \max _{|u| \leq c_{2}} F(t, u) d t-\int_{0}^{1} F\left(t, u_{1}(t)\right) d t}{r_{2}-\Phi\left(u_{1}\right)} .
$$

Making use of $\Phi\left(u_{1}\right)<r_{2}, \int_{0}^{1} \max _{|u| \leq c_{2}} F(t, u) d t-\int_{0}^{1} F\left(t, u_{1}(t)\right) d t>0$, and (3.8), we obtain

$$
\begin{aligned}
\beta\left(r_{1}, r_{2}\right) & \leq \frac{\int_{0}^{1} \max _{|u| \leq c_{2}} F(t, u) d t-\int_{0}^{1} F\left(t, u_{1}(t)\right) d t}{\frac{c_{2}^{2}}{2 M_{1}^{2}}-k_{3} c^{2}} \\
& =2 M_{1}^{2} \frac{\int_{0}^{1} \max _{|u| \leq c_{2}} F(t, u) d t-\int_{0}^{1} F\left(t, u_{1}(t)\right) d t}{c_{2}^{2}-2 k_{3} M_{1}^{2} c^{2}} \\
& =2 M_{1}^{2} a\left(c_{2}, c\right)>0 .
\end{aligned}
$$

By (2.8), and note that $\Phi\left(u_{1}\right)>r_{1}$, one has

$$
\alpha\left(r_{1}, r_{2}\right) \geq \frac{\Psi\left(u_{1}\right)-\sup _{u \in \Phi^{-1}\left(\left(-\infty, r_{1}\right)\right)} \Psi(u)}{\Phi\left(u_{1}\right)-r_{1}} .
$$

By (3.11) we have

$$
\alpha\left(r_{1}, r_{2}\right) \geq \frac{\int_{0}^{1} F\left(t, u_{1}(t)\right) d t-\int_{0}^{1} \max _{|u| \leq c_{1}} F(t, u) d t}{\Phi\left(u_{1}\right)-r_{1}} .
$$

Note that (3.7) implies that

$$
\int_{0}^{1} F\left(t, u_{1}(t)\right) d t-\int_{0}^{1} \max _{|u| \leq c_{1}} F(t, u) d t>0
$$


which, together with (3.8), gives

$$
\begin{aligned}
\alpha\left(r_{1}, r_{2}\right) & \geq \frac{\int_{0}^{1} F\left(t, u_{1}(t)\right) d t-\int_{0}^{1} \max _{|u| \leq c_{1}} F(t, u) d t}{k_{3} c^{2}-r_{1}} \\
& =2 M_{1}^{2} \frac{\int_{0}^{1} F\left(t, u_{1}(t)\right) d t-\int_{0}^{1} \max _{|u| \leq c_{1}} F(t, u) d t}{2 k_{3} M_{1}^{2} c^{2}-c_{1}^{2}} \\
& =2 M_{1}^{2} b\left(c_{1}, d\right) .
\end{aligned}
$$

Therefore, $\beta\left(r_{1}, r_{2}\right) \leq 2 M_{1}^{2} a\left(c_{2}, c\right)<2 M_{1}^{2} b\left(c_{1}, d\right) \leq \alpha\left(r_{1}, r_{2}\right)$. Thus all the conditions in [10, Theorem 5.1] are verified, and hence for each $\lambda \in\left(\lambda_{1}, \lambda_{2}\right)$ the functional $\varphi_{\lambda}=\Phi-\lambda \Psi$ admits at least one critical point $u$ such that $r_{1}<\Phi(u)<r_{2}$. Consequently, system (1.2) admits at least one solution $u$ and $\|u\|_{X}<\frac{c_{2}}{M_{1}}$.

In particular, if we take $c_{1}=0$, then (3.4) and (3.5) become

$$
a\left(c_{2}, c\right)=\frac{\int_{0}^{1} \max _{|u| \leq c_{2}} F(t, u) d t-\int_{0}^{1} F\left(t, u_{1}(t)\right) d t}{c_{2}^{2}-2 k_{3} M_{1}^{2} c^{2}}
$$

and

$$
b\left(c_{1}, c\right)=b(0, c)=\frac{\int_{0}^{1} F\left(t, u_{1}(t)\right) d t}{2 k_{3} M_{1}^{2} c^{2}} .
$$

Correspondingly, conditions (3.3) and (3.7) reduce to

$$
\sqrt{2 k_{3}} M_{1} c<c_{2}
$$

and

$$
\int_{0}^{1} \max _{|u| \leq c_{2}} F(t, u) d t<\frac{c_{2}^{2}}{2 k_{3} M_{1}^{2} c^{2}} \int_{0}^{1} F\left(t, u_{1}(t)\right) d t .
$$

If (3.13) and (3.14) hold, then

$$
\lambda_{1}=\frac{k_{3} c^{2}}{\int_{0}^{1} F\left(t, u_{1}(t)\right) d t}:=\hat{\lambda}_{1}
$$

and

$$
\begin{aligned}
\lambda_{2} & =\frac{c_{2}^{2}-2 k_{3} M_{1}^{2} c^{2}}{2 M_{1}^{2}\left(\int_{0}^{1} \max _{|u| \leq c_{2}} F(t, u) d t-\int_{0}^{1} F\left(t, u_{1}(t)\right) d t\right)} \geq \frac{c_{2}^{2}}{2 M_{1}^{2} \int_{0}^{1} \max _{|u| \leq c_{2}} F(t, u) d t} \\
& :=\hat{\lambda}_{2}>\hat{\lambda}_{1} .
\end{aligned}
$$

As a consequence, we have the following result.

Corollary 3.2 Assume that $(\mathrm{H1})$ is satisfied. If there exist two constants $c$ and $c_{2}$ satisfying (3.13) and (3.14), then for each $\lambda \in\left(\hat{\lambda}_{1}, \hat{\lambda}_{2}\right)$ system (1.2) admits at least one nontrivial solution $u$. 
Example 3.1 Consider the boundary value problem

$$
\left\{\begin{array}{l}
u^{(i v)}(t)=\lambda t, \quad t \neq t_{1}, t \in[0,1], \\
\Delta u^{\prime \prime}\left(t_{1}\right)=\frac{1}{4} u^{\prime}\left(t_{1}\right), \quad t_{1}=\frac{1}{2}, \\
-\Delta u^{\prime \prime \prime}\left(t_{1}\right)=\frac{1}{5} u\left(t_{1}\right), \quad t_{1}=\frac{1}{2}, \\
u(0)=u(1)=u^{\prime \prime}(0)=u^{\prime \prime}(1)=0 .
\end{array}\right.
$$

Here, $f(t, u)=t, I_{11}(s)=\frac{1}{4} s, I_{21}(s)=\frac{1}{5} s, A=B=0$ and $m=1$. It is easy to verify that (H1) is satisfied with $k_{1}=\frac{1}{8}$ and $k_{2}=\frac{1}{10}$. Direct calculations give $F(t, u)=t u, k_{0}=2, k_{3}=\frac{43}{20}$ and $M_{1}=1+\frac{1}{\pi} \approx 1.318$. Let $c_{1}=0.01, c=12, c_{2}=1,500$, then $c_{1}, c, c_{2}$ satisfy $(3.3)$ and $a\left(c_{2}, c\right) \approx$ $3.33 \times 10^{-4}<b\left(c_{1}, c\right) \approx 9.25 \times 10^{-4}$. Thus, it follows from Theorem 3.1 that system (3.15) has at least one solution for $\lambda \in\left(\lambda_{1}, \lambda_{2}\right)=(311.2,864.4)$.

\subsection{Existence of infinitely many solutions}

In this section, we derive some conditions under which system (1.2) admits infinitely many distinct solutions. To this end, we need the following assumptions.

(H2) Assume that

$$
\left\{t_{1}, t_{2}, \ldots, t_{m}\right\} \subseteq\left[\frac{1}{4}, \frac{3}{4}\right]
$$

(H3) Assume that

$$
F(t, u) \geq 0, \quad \text { for }(t, u) \in\left(\left[0, \frac{1}{4}\right] \cup\left[\frac{3}{4}, 1\right]\right) \times \mathbb{R} .
$$

Let $k_{4}=2,048\left(\frac{3}{8}-\frac{9}{10 \cdot 4^{4}} A+\frac{79}{14 \cdot 4^{8}} B\right)$ and $k_{5}=k_{4}+k_{2}$ with $k_{2}$ given in (3.2). We define

$$
\gamma_{1}:=\liminf _{r \rightarrow+\infty} \rho_{1}(r), \quad \gamma_{2}:=\liminf _{r \rightarrow\left(\inf _{X} \Phi\right)^{+}} \rho_{1}(r),
$$

where $\rho_{1}(r)$ is given $(2.10)$. Let

$$
\mu_{1}=2 M_{1}^{2} \liminf _{\xi \rightarrow+\infty} \frac{\int_{0}^{1} \max _{|u| \leq \xi} F(t, u) d t}{\xi^{2}}, \quad \mu_{2}=\frac{1}{k_{5}} \limsup _{\xi \rightarrow+\infty} \frac{\int_{\frac{1}{4}}^{\frac{3}{4}} F(t, \xi) d t}{\xi^{2}} .
$$

Theorem 3.3 Assume that $(\mathrm{H} 1),(\mathrm{H} 2)$, and $(\mathrm{H} 3)$ are satisfied. If

$$
\mu_{1}<\mu_{2}
$$

holds, then for each $\lambda \in\left(\frac{1}{\mu_{2}}, \frac{1}{\mu_{1}}\right)$ system (1.2) has an unbounded sequence of solutions in $X$.

Proof We apply [5, Theorem 2.1] to show that the functional $\varphi_{\lambda}$ defined in (2.3) has an unbounded sequence of critical points.

We first show that $\gamma_{1}<+\infty$. Let $\left\{\xi_{n}\right\}$ be a sequence of positive numbers such that $\xi_{n} \rightarrow$ $+\infty$ as $n \rightarrow \infty$ and

$$
\lim _{n \rightarrow+\infty} \frac{\int_{0}^{1} \max _{|u| \leq \xi n} F(t, u) d t}{\xi_{n}^{2}}=\liminf _{\xi \rightarrow+\infty} \frac{\int_{0}^{1} \max _{|u| \leq \xi} F(t, \xi) d t}{\xi^{2}} .
$$


For any positive integer $n$, we let $r_{n}=\frac{\xi_{n}^{2}}{2 M_{1}^{2}}$. For $u \in X$ satisfying $\Phi(u)<r_{n}$, similar to the proof of Theorem 3.1, one can show that

$$
\|u\|_{\infty}^{2} \leq 2 M_{1}^{2} \Phi(u)<\xi_{n}^{2}, \quad t \in[0,1]
$$

which implies that

$$
\Psi(u)=\int_{0}^{1} F(t, u) d t \leq \int_{0}^{1} \max _{|u| \leq \xi_{n}} F(t, u) d t .
$$

Note that $\Psi(0)=\Phi(0)=0$, thus we have

$$
\begin{aligned}
\rho_{1}\left(r_{n}\right) & =\inf _{v \in \Phi^{-1}\left(\left(-\infty, r_{n}\right)\right)} \frac{\sup _{u \in \Phi^{-1}\left(\left(-\infty, r_{n}\right)\right)} \Psi(u)-\Psi(v)}{r_{n}-\Phi(v)} \\
& \leq \frac{\sup _{u \in \Phi^{-1}\left(\left(-\infty, r_{n}\right)\right)} \Psi(u)-\Psi(0)}{r_{n}-\Phi(0)} \\
& =\frac{\sup _{u \in \Phi^{-1}\left(\left(-\infty, r_{n}\right)\right)} \Psi(u)}{r_{n}} \\
& \leq \frac{2 M_{1}^{2} \int_{0}^{1} \max _{|u| \leq \xi_{n}} F(t, u) d t}{\xi_{n}^{2}},
\end{aligned}
$$

which, together with (3.16), gives us

$$
\gamma_{1} \leq 2 M_{1}^{2} \liminf _{\xi \rightarrow+\infty} \frac{\int_{0}^{1} \max _{|u| \leq \xi} F(t, u) d t}{\xi^{2}}=\mu_{1}<+\infty .
$$

This shows that $\left(\frac{1}{\mu_{2}}, \frac{1}{\mu_{1}}\right) \subseteq\left(0, \frac{1}{\gamma_{1}}\right)$. For any fixed $\lambda \in\left(\frac{1}{\mu_{2}}, \frac{1}{\mu_{1}}\right)$, it follows from [5, Theorem 2.1] that either $\varphi_{\lambda}=\Phi-\lambda \Psi$ has a global minimum or there is a sequence $\left\{u_{n}\right\}$ of critical points (local minima) of $\varphi_{\lambda}$ such that $\lim _{n \rightarrow+\infty}\left\|u_{n}\right\|_{X}=+\infty$.

Next we show that the functional $\varphi_{\lambda}$ has no global minimum for $\lambda \in\left(\frac{1}{\mu_{2}}, \frac{1}{\mu_{1}}\right)$. Since $\lambda>$ $\frac{1}{\mu_{2}}=k_{5} / \lim \sup _{\xi \rightarrow+\infty} \frac{\int_{\frac{1}{4}}^{\frac{3}{4}} F(t, \xi) d t}{\xi^{2}}$, we can choose a constant $M$ such that, for each $n \in N=$ $\{1,2, \ldots\}$,

$$
\sup _{\xi \geq n} \frac{\int_{\frac{1}{4}}^{\frac{3}{4}} F(t, \xi) d t}{\xi^{2}}>M>\frac{k_{5}}{\lambda} .
$$

Thus, there exists $\xi_{n} \geq n$ such that

$$
\frac{\int_{\frac{1}{4}}^{\frac{3}{4}} F\left(t, \xi_{n}\right) d t}{\xi_{n}^{2}}>M .
$$

Define $u_{n}(t)$ as follows:

$$
u_{n}(t)= \begin{cases}64 \xi_{n}\left(t^{3}-\frac{3}{4} t^{2}+\frac{3}{16} t\right), & t \in\left[0, \frac{1}{4}\right), \\ \xi_{n}, & t \in\left[\frac{1}{4}, \frac{3}{4}\right], \\ 64 \xi_{n}\left(-t^{3}+\frac{9}{4} t^{2}-\frac{27}{16} t+\frac{7}{16}\right), & t \in\left(\frac{3}{4}, 1\right] .\end{cases}
$$


This, together with (H2), yields

$$
\begin{aligned}
\Phi\left(u_{n}\right) & =2,048\left(\frac{3}{8}-\frac{9}{10 \cdot 4^{4}} A+\frac{79}{14 \cdot 4^{8}} B\right) \xi_{n}^{2}+\sum_{j=1}^{m} \int_{0}^{\xi_{n}} I_{2 j}(s) d s \\
& \leq k_{4} \xi_{n}^{2}+k_{2} \xi_{n}^{2} \\
& =k_{5} \xi_{n}^{2} .
\end{aligned}
$$

It then follows from $(\mathrm{H} 3)$ that

$$
\begin{aligned}
\varphi_{\lambda}\left(u_{n}\right) & =\Phi\left(u_{n}\right)-\lambda \Psi\left(u_{n}\right) \\
& \leq k_{5} \xi_{n}^{2}-\lambda \int_{\frac{1}{4}}^{\frac{3}{4}} F\left(t, \xi_{n}\right) d t \\
& \leq \xi_{n}^{2}\left(k_{5}-\lambda M\right) .
\end{aligned}
$$

Note that $k_{5}-\lambda M<0$. Thus the functional $\varphi_{\lambda}$ is unbounded from below and hence it has no global minimum and the proof is complete.

Corollary 3.4 Assume that (H1), (H2), and (H3) are satisfied.If

$$
\liminf _{\xi \rightarrow+\infty} \frac{\int_{0}^{1} \max _{|u| \leq \xi} F(t, u) d t}{\xi^{2}}<\frac{1}{2 M_{1}^{2}}
$$

and

$$
\limsup _{\xi \rightarrow+\infty} \frac{\int_{\frac{1}{4}}^{\frac{3}{4}} F(t, \xi) d t}{\xi^{2}}>k_{5}
$$

hold, then (1.2) has an unbounded sequence of solutions in X.

Let

$$
\mu_{3}=2 M_{1}^{2} \liminf _{\omega \rightarrow 0^{+}} \frac{\int_{0}^{1} \max _{|u| \leq \omega} F(t, u) d t}{\omega^{2}}, \quad \mu_{4}=\frac{1}{k_{5}} \limsup _{\omega \rightarrow 0^{+}} \frac{\int_{\frac{1}{4}}^{\frac{3}{4}} F(t, \omega) d t}{\omega^{2}} .
$$

Theorem 3.5 Assume that (H1), (H2), and (H3) are satisfied. If

$$
\mu_{3}<\mu_{4}
$$

holds, then for each $\lambda \in\left(\frac{1}{\mu_{4}}, \frac{1}{\mu_{3}}\right)$ system (1.2) has a sequence of non-zero solutions in $X$, which weakly converges to 0 .

Proof The proof is similar to that of Theorem 3.3 by showing that $\gamma_{2}<+\infty$ and 0 is not a local minimum of the functional $\varphi_{\lambda}=\Phi-\lambda \Psi$. 
Example 3.2 Consider

$$
\left\{\begin{array}{l}
u^{(i v)}(t)-2 u^{\prime \prime}(t)+u=\lambda f(t, u), \quad t \neq t_{1}, t \in[0,1] \\
\Delta u^{\prime \prime}\left(t_{1}\right)=\frac{1}{10} u^{\prime}\left(t_{1}\right), \quad t_{1}=\frac{1}{2} \\
-\Delta u^{\prime \prime \prime}\left(t_{1}\right)=\frac{1}{20} u\left(t_{1}\right), \quad t_{1}=\frac{1}{2} \\
u(0)=u(1)=u^{\prime \prime}(0)=u^{\prime \prime}(1)=0
\end{array}\right.
$$

where $f(t, u)=4 t u(1+\sin u)+2 t u^{2} \cos u$

Here $I_{11}(s)=\frac{1}{10} s, I_{21}(s)=\frac{1}{20} s, A=-2, B=1$ and $m=1$. Note that

$$
\begin{aligned}
& \int_{0}^{u^{\prime}\left(t_{1}\right)} I_{11}(s) d s=\int_{0}^{u^{\prime}\left(t_{1}\right)} \frac{1}{10} s d s=\frac{1}{20}\left|u^{\prime}\left(t_{1}\right)\right|^{2}, \\
& \int_{0}^{u\left(t_{1}\right)} I_{21}(s) d s=\int_{0}^{u\left(t_{1}\right)} \frac{1}{20} s d s=\frac{1}{40}\left|u\left(t_{1}\right)\right|^{2}, \\
& t_{1}=\frac{1}{2} \in\left[\frac{1}{4}, \frac{3}{4}\right] \\
& F(t, u)=2 t(1+\sin u) u^{2},
\end{aligned}
$$

so $(\mathrm{H} 1),(\mathrm{H} 2)$, and $(\mathrm{H} 3)$ are satisfied. Moreover, we have $k_{5} \approx 782.6$, and

$$
\lim _{\xi \rightarrow+\infty} \inf \frac{\int_{0}^{1} \max _{|u| \leq \xi} F(t, u) d t}{\xi^{2}}=0, \quad \lim _{\xi \rightarrow+\infty} \sup \frac{\int_{\frac{1}{4}}^{\frac{3}{4}} F(t, \xi) d t}{\xi^{2}}=1 .
$$

Therefore, condition (3.17) holds and Theorem 3.3 applies: For $\lambda \in(782.6,+\infty),(3.19)$ admits an unbounded sequence of solutions in $X$.

Example 3.3 Consider the boundary value problem

$$
\left\{\begin{array}{l}
u^{(i v)}(t)-2 u^{\prime \prime}(t)+u=\lambda f(t, u), \quad t \neq t_{1}, t \in[0,1] \\
\Delta u^{\prime \prime}\left(t_{1}\right)=\frac{1}{5} u^{\prime}\left(t_{1}\right), \quad t_{1}=\frac{1}{2} \\
-\Delta u^{\prime \prime \prime}\left(t_{1}\right)=\frac{1}{8} u\left(t_{1}\right), \quad t_{1}=\frac{1}{2} \\
u(0)=u(1)=u^{\prime \prime}(0)=u^{\prime \prime}(1)=0
\end{array}\right.
$$

where

$$
f(t, u(t))= \begin{cases}4 t u\left(0.5001+\frac{1}{2} \cos (\ln |u|)-\frac{1}{4} \sin (\ln (|u|))\right) & \text { if } u \neq 0 \\ 0 & \text { if } u=0\end{cases}
$$

In this example, $I_{11}(s)=\frac{1}{5} s, I_{21}(s)=\frac{1}{8} s, A=-2$ and $B=1$. The assumptions (H1), (H2), and (H3) clearly hold.

Direct calculations give

$$
F(t, u(t))= \begin{cases}2 t u^{2}\left(0.5001+\frac{1}{2} \cos (\ln |u|)\right) & \text { if } u \neq 0 \\ 0 & \text { if } u=0\end{cases}
$$


$k_{5} \approx 762.64$ and

$$
\lim _{w \rightarrow 0^{+}} \inf \frac{\int_{0}^{1} \max _{|u| \leq w} F(t, u) d t}{w^{2}}=0.0001,
$$

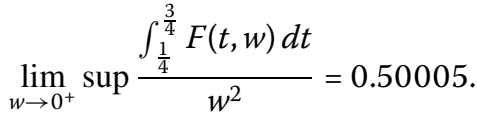

Hence (3.18) holds. Therefore it follows from Theorem 3.5 that (3.20) admits a sequence of distinct solutions in $X$ provided that $\lambda \in(1525.1,2877.0)$.

\section{Competing interests}

The authors declare that they have no competing interests.

\section{Authors' contributions}

Both authors made equal contribution. Both authors read and approved the final manuscript.

\section{Author details}

${ }^{1}$ College of Mathematics and Statistics, Jishou University, Jishou, Hunan 416000, P.R. China. ${ }^{2}$ Department of Mathematics, Hunan Normal University, Changsha, Hunan 410081, P.R. China.

\section{Authors' information}

JX is with the College of Mathematics and Statistics, Jishou University, China and is a PhD candidate at the Department of Mathematics, Hunan Normal University, China. ZL is a professor at the Department of Mathematics, Hunan Normal University, China.

\section{Acknowledgements}

The authors are very grateful to the referees for their valuable comments and suggestions, which greatly improved the presentation of this paper. The work is partially supported by Hunan Provincial Natural Science Foundation of China (No: 11JJ3012).

Received: 26 March 2013 Accepted: 15 June 2013 Published: 1 July 2013

\section{References}

1. Aftabizadeh, AR: Existence and uniqueness theorems for fourth-order boundary value problems. J. Math. Anal. Appl. $116,415-426(1986)$

2. Gupta, CP: Existence and uniqueness theorems for the bending of an elastic beam equation. Appl. Anal. 26, 289-304 (1988)

3. Ma, R, Zhan, J, Fu, S: The method of lower and upper solutions for fourth-order two-point boundary value problems. J. Math. Anal. Appl. 215, 415-422 (1997)

4. Bonanno, G, Dibella, B, Regan, DO: Non-trivial solutions for nonlinear fourth-order elastic beam equations. Comput. Math. Appl. 62, 1862-1869 (2011)

5. Bonanno, G, Molica Bisci, G: Infinitely many solutions for a boundary value problem with discontinuous nonlinearities. Bound. Value Probl. 113, 1-20 (2009)

6. Han, G, Xu, Z: Multiple solutions of some nonlinear fourth-order beam equations. Nonlinear Anal. 68, 3646-3656 (2008)

7. Liu, X, Li, W: Existence and multiplicity of solutions for fourth-order boundary values problems with parameters. J. Math. Anal. Appl. 327, 362-375 (2007)

8. Agarwal, RP, Rgean, DO: A multiplicity result for second order impulsive differential equations via the Leggett Williams fixed point theorem. Appl. Math. Comput. 161, 433-439 (2005)

9. Agarwal, RP, Franco, D, Regan, DO: Singular boundary value problems for first and second order impulsive differential equations. Aequ. Math. 69, 83-96 (2005)

10. Bonanno, G: A critical point theorem via the Ekeland variational principle. Nonlinear Anal. 75, 2992-3007 (2012)

11. Chen, J, Nieto, JJ: Impulsive periodic solutions of first-order singular differential equations. Bull. Lond. Math. Soc. 40, 902-912 (2007)

12. De Coster, C, Habets, P: Upper and lower solutions in the theory of ODE boundary value problems: classical and recent results. In: Zanolin, F (ed.) Nonlinear Analysis and Boundary Value Problem for Ordinary Differential Equations CISM-ICMS, vol. 371, pp. 1-78. Springer, New York (1996)

13. Marek, G, Szymon, G: On the discrete boundary value problem for anisotropic equation. J. Math. Anal. Appl. 386 956-965 (2012)

14. Nieto, JJ, Regan, DO: Variational approach to impulsive differential equations. Nonlinear Anal., Real World Appl. 10 680-690 (2009)

15. Qin, D, Li, X: Periodic solutions for ordinary differential equations with sublinear impulsive effects. J. Math. Anal. Appl. 303, 288-303 (2005)

16. Shen, JH, Wang, BW: Impulsive boundary value problems with nonlinear boundary conditions. Nonlinear Anal. 69 4055-4062 (2008)

17. Tian, Y, Ge, W: Applications of variational methods to boundary-value problem for impulsive differential equations. Proc. Edinb. Math. Soc. 51, 509-527 (2008)

18. Zhang, H, Li, Z: Variational approach to impulsive differential equations with periodic boundary conditions. Nonlinear Anal., Real World Appl. 11, 67-78 (2010) 
19. Zhang, $X$, Tang, $X$ : Subharmonic solutions for a class of non-quadratic second order Hamiltonian systems. Nonlinear Anal., Real World Appl. 13, 113-130 (2012)

20. Zhang, Z, Yuan, R: Applications of variational methods to Dirichlet boundary value problem with impulses. Nonlinear Anal., Real World Appl. 11, 155-162 (2010)

21. Sun, J, Chen, H, Yang, L: Variational methods to fourth-order impulsive differential equations. J. Appl. Math. Comput. 35, 323-340 (2011)

22. Dym, H, McKean, H: Fourier Series and Integrals. Academic Press, New York (1985)

23. Peletier, LA, Troy, WC, van der Vorst, RCAM: Stationary solutions of a fourth order nonlinear diffusion equation. Differ. Equ. 31, 301-314 (1995)

doi:10.1186/1687-2770-2013-154

Cite this article as: Xie and Luo: Solutions to a boundary value problem of a fourth-order impulsive differential equation. Boundary Value Problems 2013 2013:154

Submit your manuscript to a SpringerOpen ${ }^{\circ}$ journal and benefit from:

- Convenient online submission

- Rigorous peer review

- Immediate publication on acceptance

- Open access: articles freely available online

- High visibility within the field

- Retaining the copyright to your article 